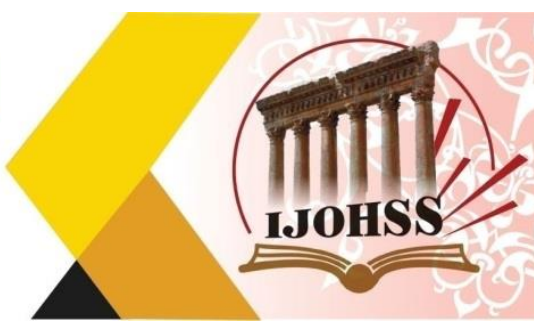

\title{
دراسة التغير النسبي لقيم الاوزون في الموصل 1
}

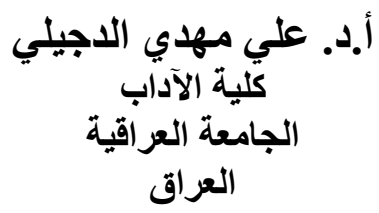

ali.al-dujaili@aliraqia.edu.iq البريد الاكتتروني:

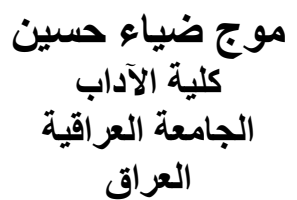

jojaia.dh@gmail.com البريد الاكتروني:

|لماخص

يعد علم المناخ من العلوم التطبيقية التي قلما نجد منافسا له في مجال ارتباطه الوثيق بحياة الانسان ومظاهر

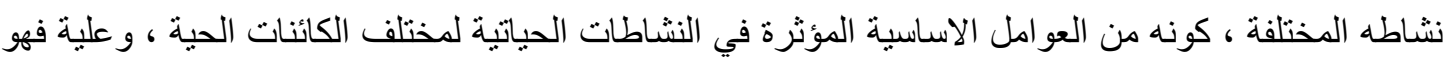
يمكن الباحثين من فهم مختلف الظو اهر الحيوية وتفسير ها بعد فهم محيطها ، وبالتالي وضع استر اتيجيات ، تكون بمثابة واق للبيئة من خطر التدهور و التلوث ، و عند ذللك يكون قد حافظ على الحباة الطبيعية ـ يرمي البحث الى تحديد قيم الأوزون الكلية في العراق ، من خلال استخدام الطرق الاحصائية واختيار أهم الطر ائق التي يمكن من خلالها حساب الأوزون في منطقة الدر اسة ، وبالتالي تحليل التغير النسبي في منطقة الدراسة تم تطبيق هذا البحث في محطة (الموصل) ، لذ سوف يتم في هذا الفصل الاعتماد على استخدام أسلوب الاتجاه العام ومعدل التغير النسبي من اجل إيضاح التغيرات الحاصلة في قيم الأوزون بمنطقة الدراسة، في وللكثف عن الاتجاه العام ومعدل التغير منطقة الدر اسة (Trend Detection) تم حساب الاتجاه العام للمعدلات السنوية للسلاسل الزمنية (لعناصر المناخ)، وتم التعبير عن معامل الاتجاه بالنسبة المئوية لمجمل المتغيرات في عناصر المناخ. . 


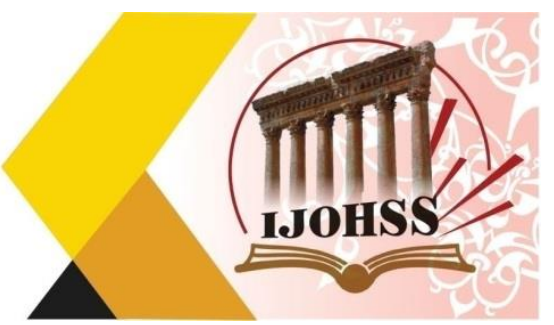

\title{
Study of the Relative Change of Ozone Values in Mosul
}

\author{
Mauj Diaa Hussein \\ College of Arts \\ Al-Iraqiya University \\ Iraq \\ Email: jojaia.dh@gmail.com
}

\author{
Prof. Dr. Ali Mahdi Al-Dujaili \\ College of Arts \\ Al-Iraqiya University \\ Iraq \\ Email: ali.al-dujaili@aliraqia.edu.iq
}

\begin{abstract}
Climatology is one of the applied sciences that we rarely find a competitor in in the field of its close connection with human life and the various manifestations of its activity, as it is one of the main factors affecting the life activities of various living organisms, and therefore it enables researchers to understand and explain various vital phenomena after understanding their surroundings, and thus develop strategies It serves as a safeguard for the environment from the danger of deterioration and pollution, and then it has preserved normal life. The research aims to determine the total ozone values in Iraq, through the use of statistical methods and the selection of the most important methods through which the ozone can be calculated in the study area, and thus analyze the relative change in the study area. This research was applied in the station (Mosul), so it will be done in this The chapter is based on the use of the general trend method and the relative rate of change in order to clarify the changes occurring in the ozone values in the study area, and to reveal the general trend and rate of change in the study area (Trend Detection). The trend coefficient as a percentage of all the variables in the climate elements.
\end{abstract}

Keywords: relative change, ozone values, Mosul city. 


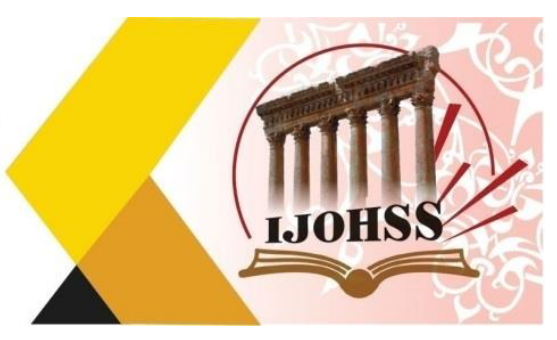

Introduction المقدمة

تعني كلمة أوزون باللغة اللاتينية رائحة ( odor ) ، و ويعد غاز الأوزون احد مكونات الغلاف الجوي المهمة .

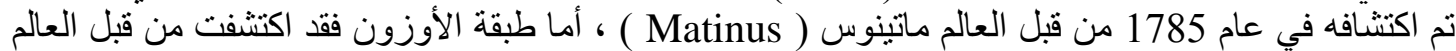

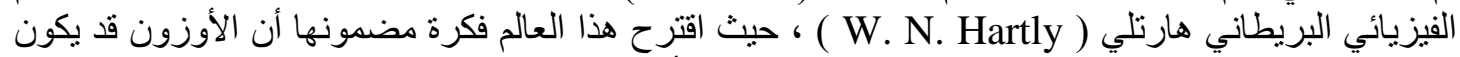

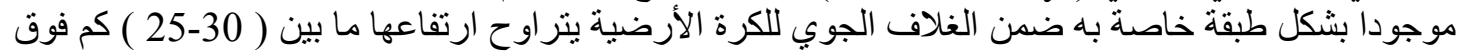

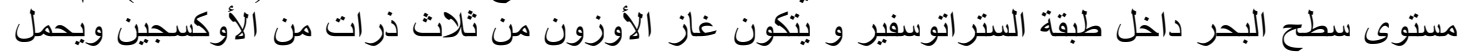

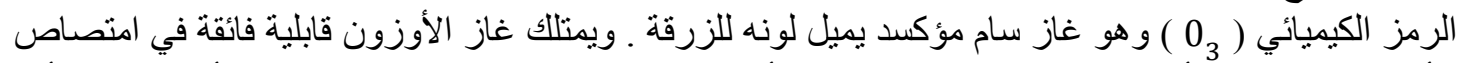

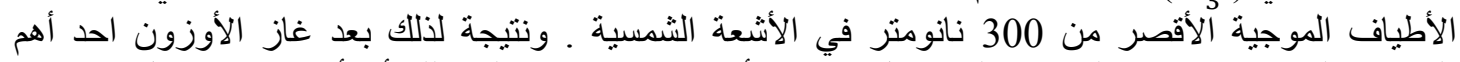

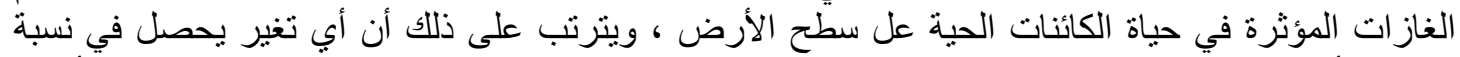

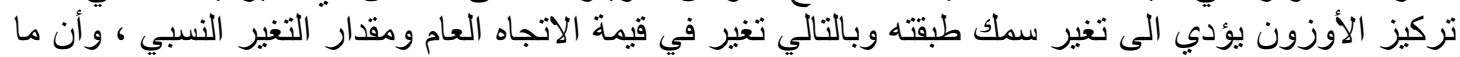

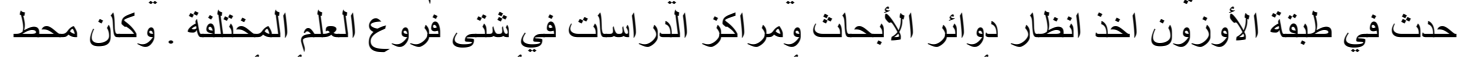

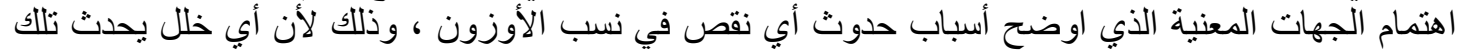

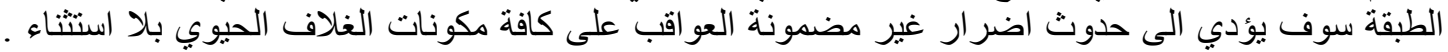

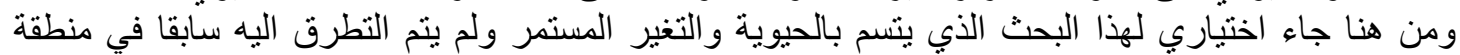

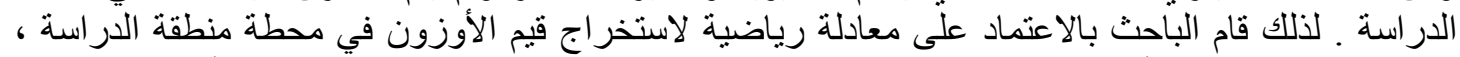

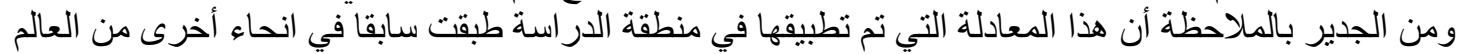

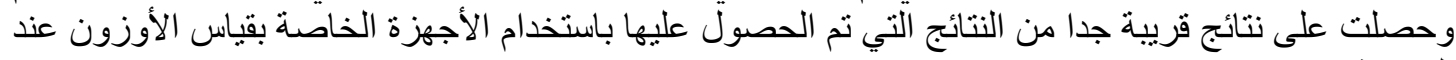
المقارنة.

مشكلة الاراسة: Study Problem (هل يوجد تغير نسبي لقيم الاوزون في الموصل؟)

فرضية البحث: Rypothesis ولغرض الاجابة عن سؤ ال مشكلة البحث فان ذلك ينطلب وضع فرضية للبحث فتتلخص بما يلي: (نعم يوجد تغير نسبي لقيم الاوزون في الموصل.)

اهمية البحث: Importance of research

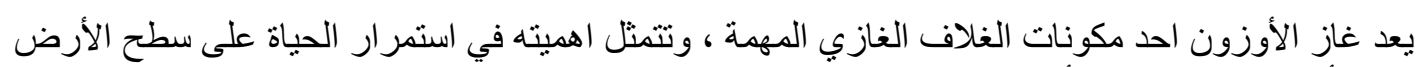

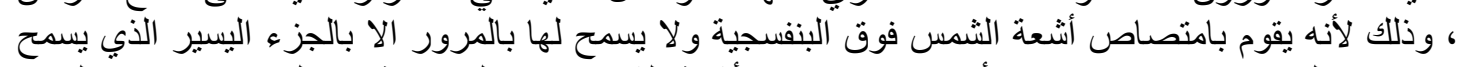

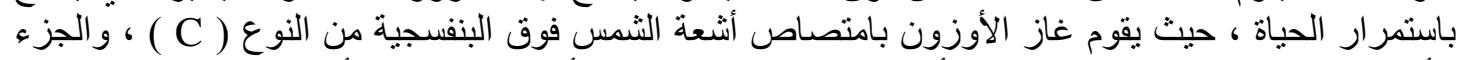

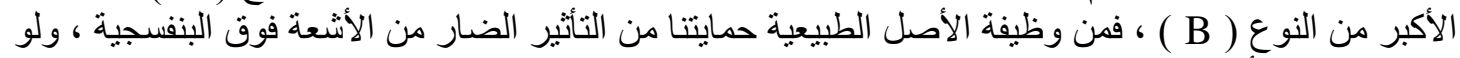

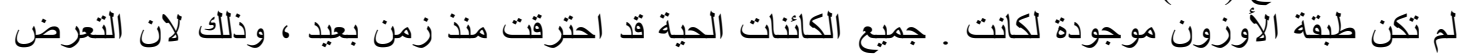

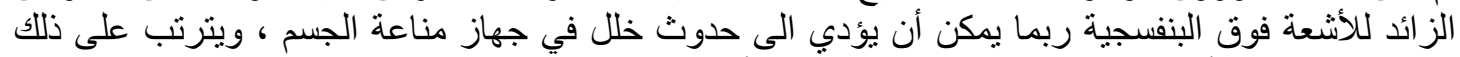

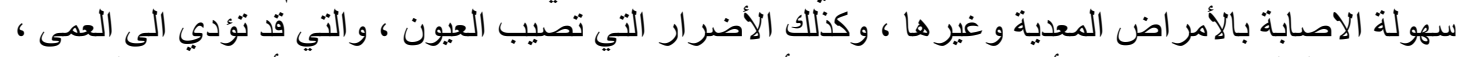

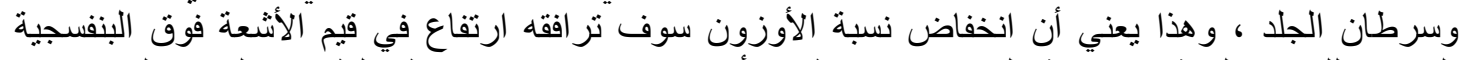

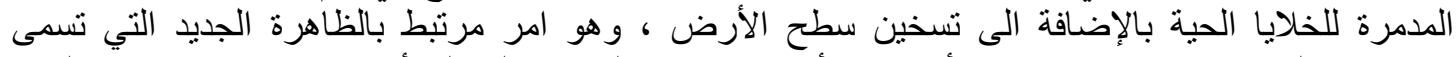

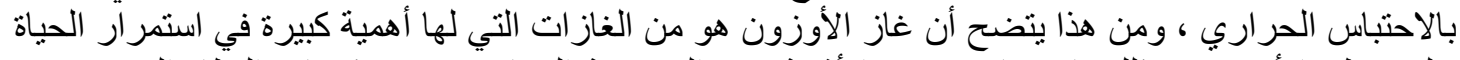

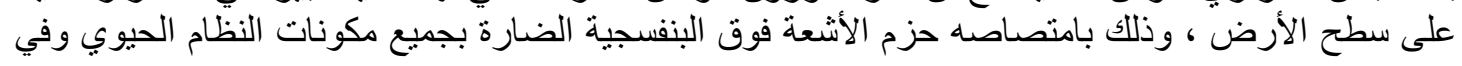




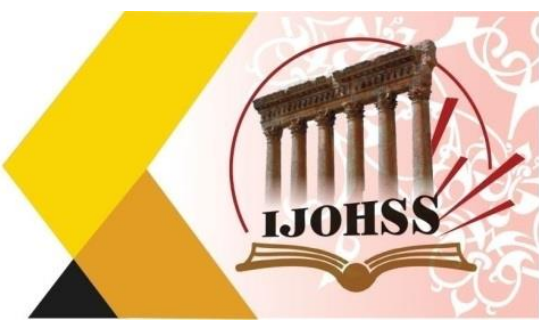

مقدمتها الانسان ، ولهذا فمن الضروري حساب قيم الأوزون في منطقة الدراسة ، ولعدم وجود دراسات سابقة

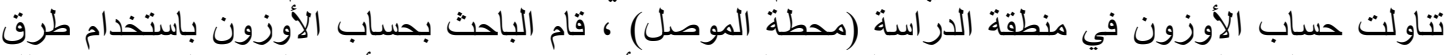

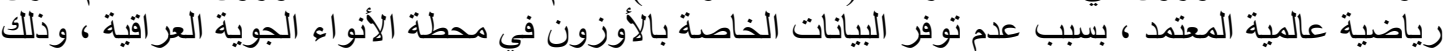

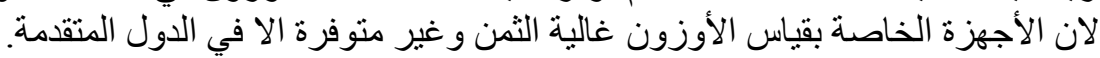

هesearch Objective : هدف البحث

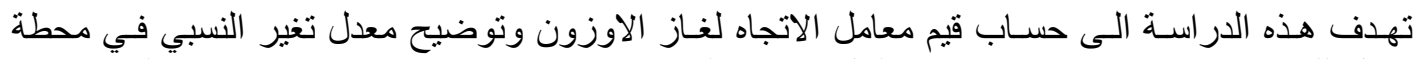

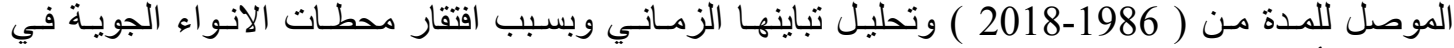
العـر اق لأجهزة قيـاس نفاذيـة غـاز الاوزون ، قامت الباحثة بالاستعاضة عن ذلك بالمعادلات الرياضية.

حدود منطقة الدراسة: Boundaries Of The Study Area

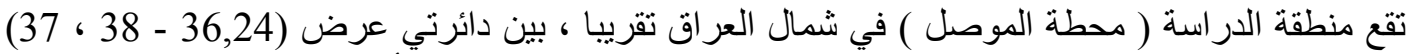

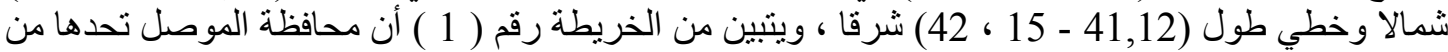

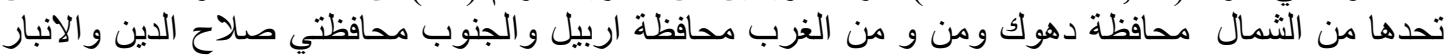

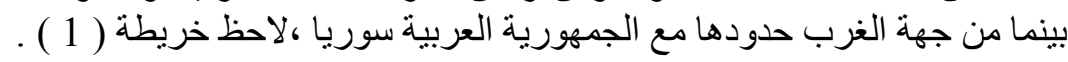

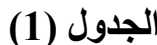

الموقع القلكي لمنطقة الارلة (1) - اسة

\begin{tabular}{|c|c|c|c|c|}
\hline رقم المحطة & خطوط الطول & دو ائر العرض & الارتفاع بالأمتار & المحطة \\
\hline 608 & 43.09 & 36.32 & 223 & الموصل \\
\hline
\end{tabular}

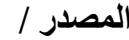

جمهورية العراق ، وزارة النقل والمواصلات ، الهيئة العامة للأنواء الجوية والرصد الزلزالي ، اطلس مناخ العراق (

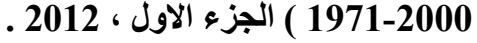


المجلة الدولية اللملوم الإنسانية والإمتصاعية International Journal on Humanities and Social Sciences website:www.ijohss.com Email:editor@ijohss.com العدد (26) أكتوبر 2021 ISSN: $2415-\mathbf{4 8 2 2}$

Volume (26) October 2021

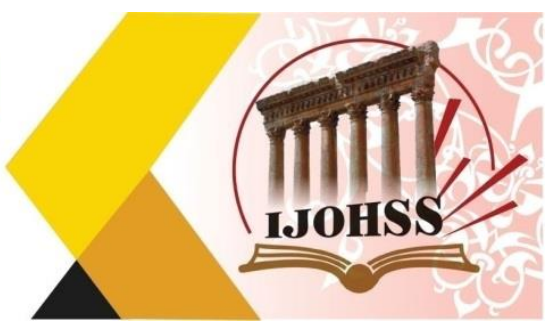

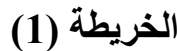

موقع محطة موصل

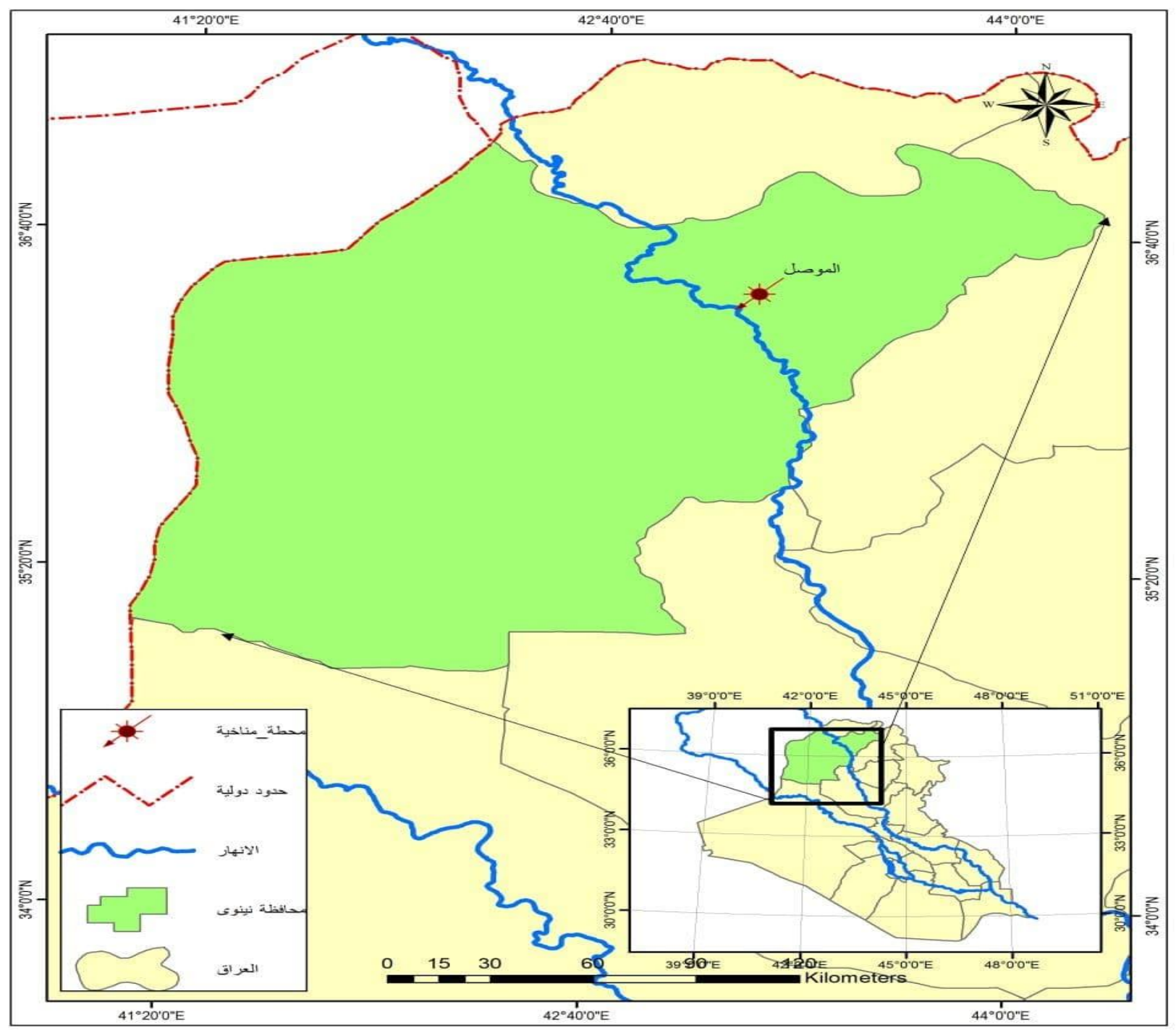

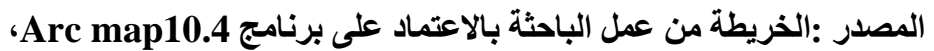

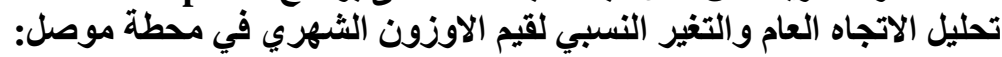

Analysis of the general trend and the relative change of monthly ozone values in the Mosul station:

ويلاحظ من جدول (2) وشكل (1) خلال اشهر السنة قد سجل محطة الموصل في مدة الدراسة اعلى معامل

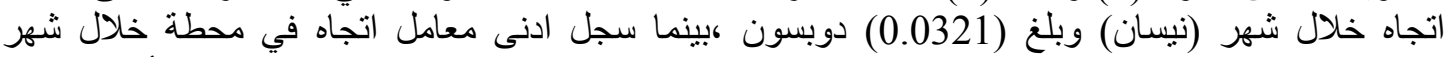

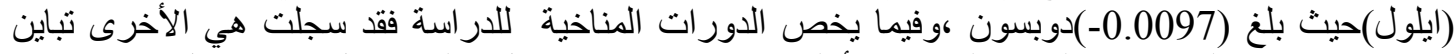

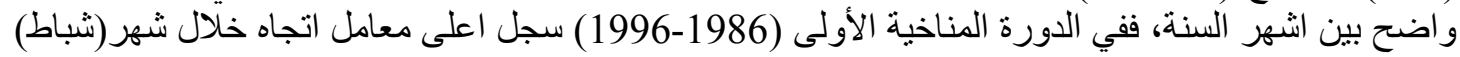

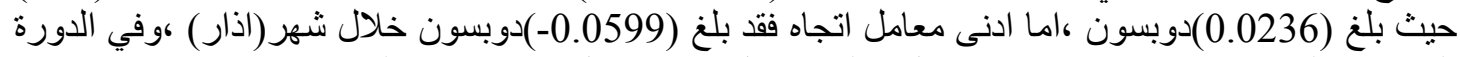

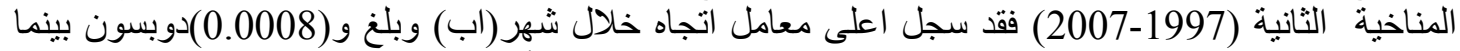
سجل ادنى معامل اتجاه خلال شهر(شباط) وبلغ (2319.0.23-)دوبسون ،و أخذ بعد ذلك معامل الاتجاه بالتغير خلال 
International Journal on Humanities and Social Sciences

website:www.ijohss.com

Email:editor@ijohss.com

العدد (26) أكتوبر 2021

ISSN: $2415-4822$

Volume (26) October 2021

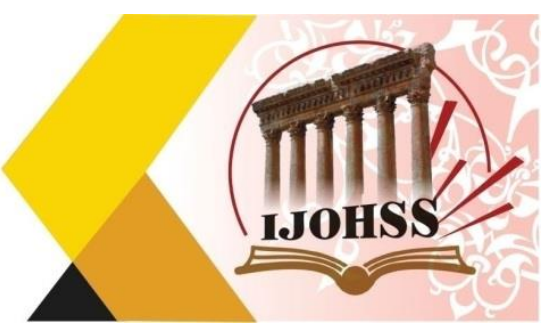

الدورة المناخية الثالثة (2008-2018) حيث انتقل في جميع الاشهر المدروسة من الاتجاه السالب الى الاتجاه

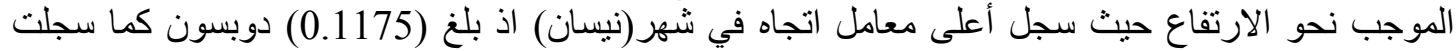

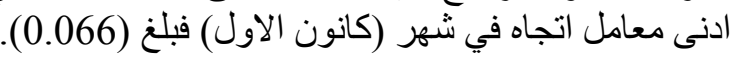

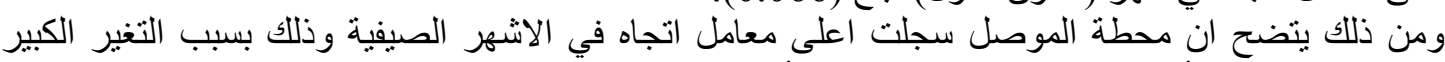

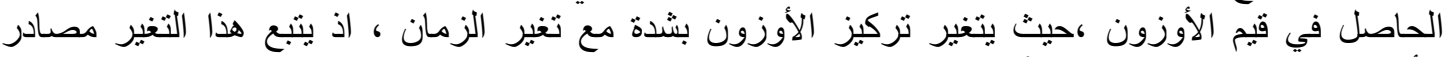

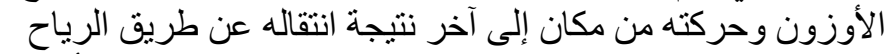

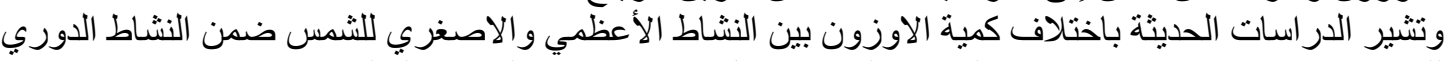

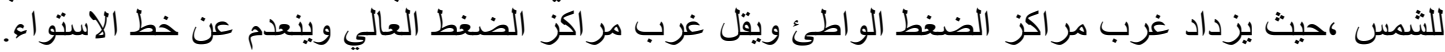

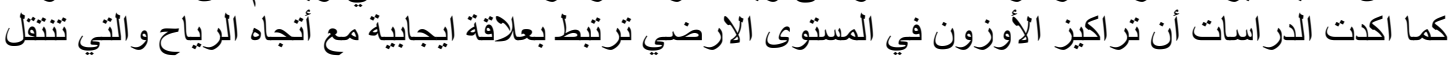

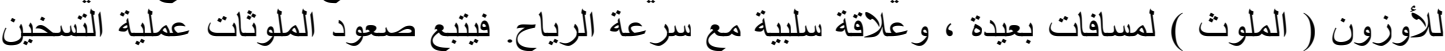

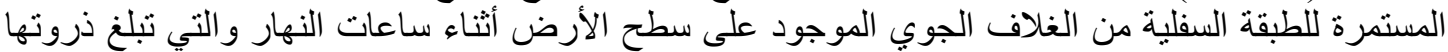

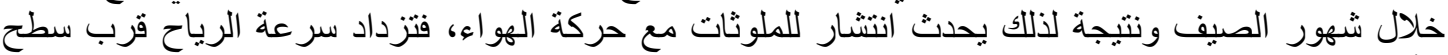

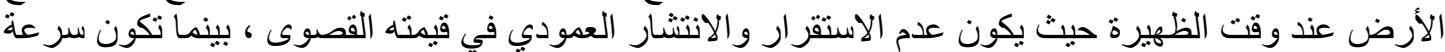

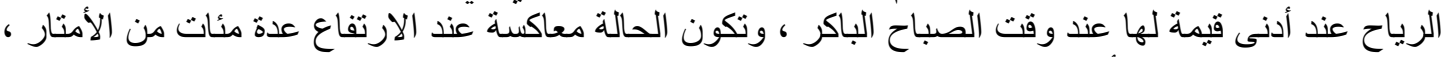

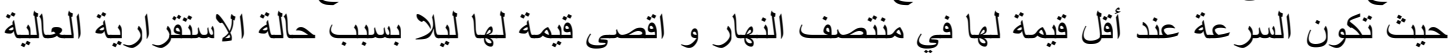

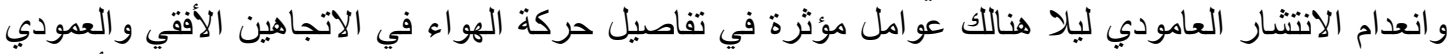

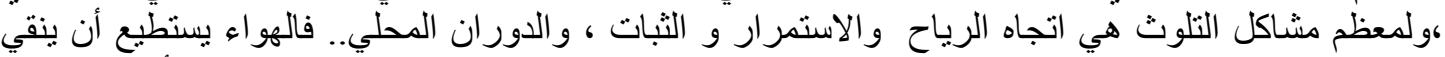

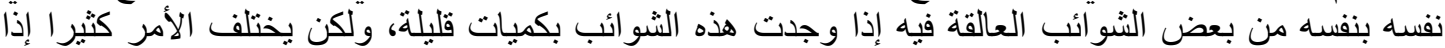

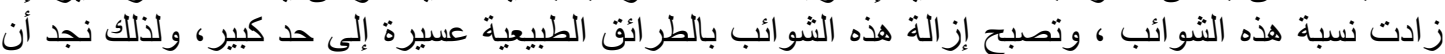

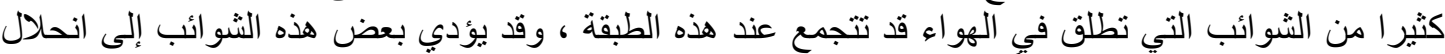

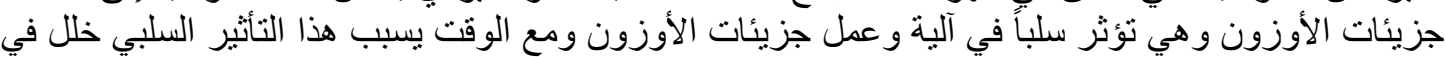

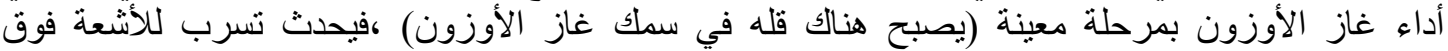

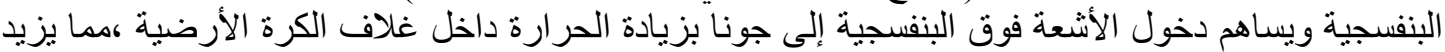

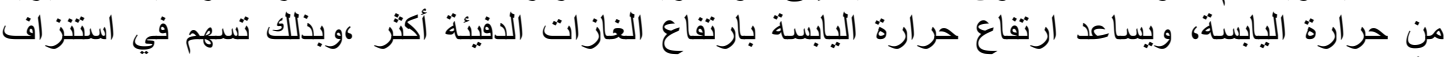

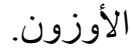

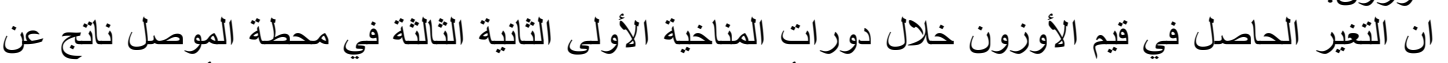

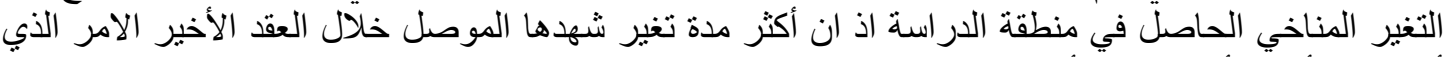

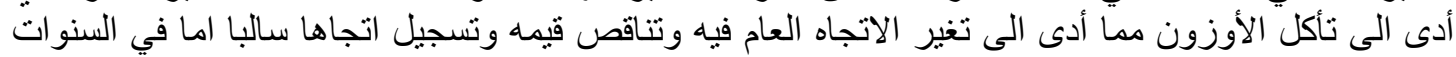

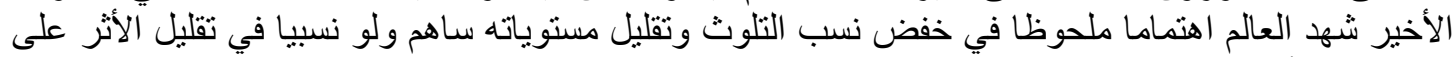

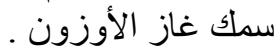

اما بالنسبة للتغير النسبي الحاصل في قيم الأوزون فقد شهد تغيرات خلال المدة الدراسة في محطة الموصل

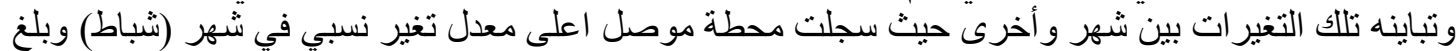

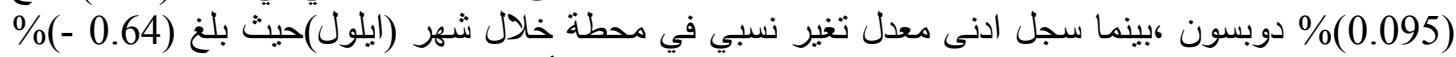

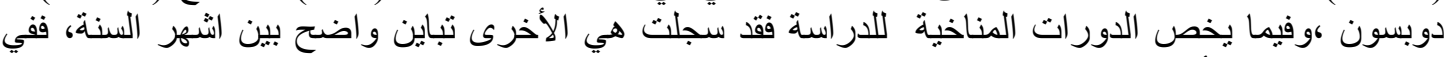

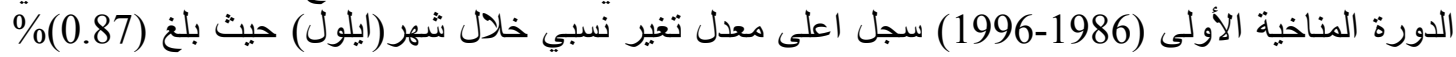

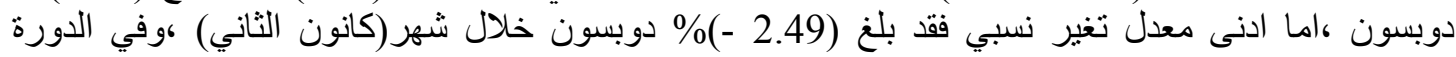

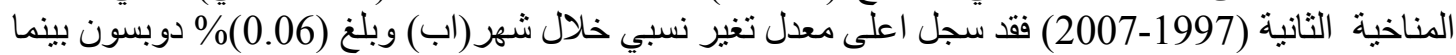

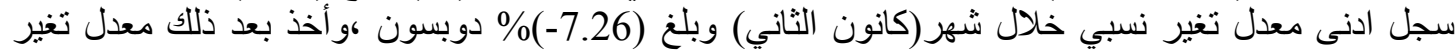

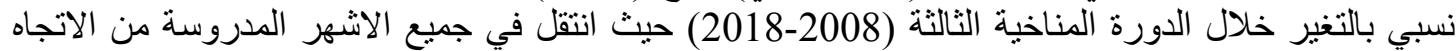

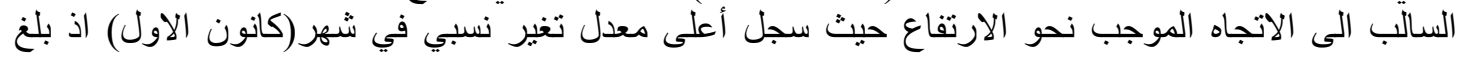

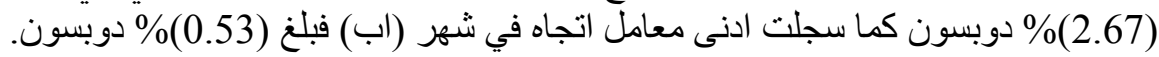




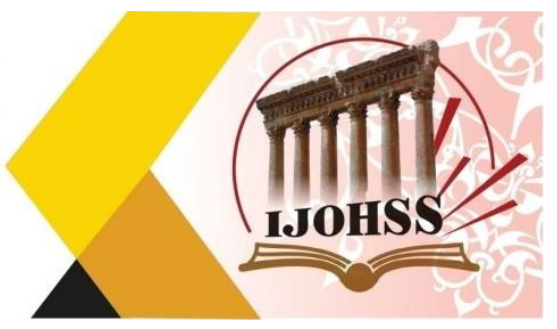

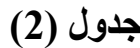

معامل الاتجاه والتغير لقيم الاوزون لأشهر السنة للمدة من (1986-2018) في محطة الموصل

\begin{tabular}{|c|c|c|c|c|c|}
\hline معدل التغير النسبي & الاتجاه & المتوسط & السنوات & مدة السلسلة & الاشهر \\
\hline-0.03 & -0.001 & 3.34 & 33 & $2018-1986$ & \multirow{4}{*}{$\begin{array}{l}\frac{y}{3} \\
.3 \\
\sqrt[3]{3}\end{array}$} \\
\hline-2.49 & -0.080 & 3.21 & 11 & $1996-1986$ & \\
\hline-7.26 & -0.246 & 3.39 & 11 & $2007-1997$ & \\
\hline 1.92 & 0.066 & 3.43 & 11 & $2018-2008$ & \\
\hline 0.095 & 0.0038 & 3.97 & 33 & $2018-1986$ & \multirow{4}{*}{ 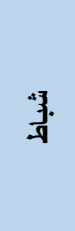 } \\
\hline 0.60 & 0.0236 & 3.94 & 11 & $1996-1986$ & \\
\hline-6.09 & -0.2319 & 3.81 & 11 & $2007-1997$ & \\
\hline 1.97 & 0.0818 & 4.15 & 11 & $2018-2008$ & \\
\hline-0.016 & -0.0007 & 4.29 & 33 & $2018-1986$ & \multirow{4}{*}{ 高 } \\
\hline-1.43 & -0.0599 & 4.19 & 11 & $1996-1986$ & \\
\hline-4.99 & -0.2155 & 4.32 & 11 & $2007-1997$ & \\
\hline 1.88 & 0.0819 & 4.35 & 11 & $2018-2008$ & \\
\hline 0.77 & 0.0321 & 4.12 & 33 & $2018-1986$ & \multirow{4}{*}{ 沀 } \\
\hline-0.33 & -0.0120 & 3.61 & 11 & $1996-1986$ & \\
\hline-2.81 & -0.1218 & 4.33 & 11 & $2007-1997$ & \\
\hline 2.66 & 0.1175 & 4.42 & 11 & $2018-2008$ & \\
\hline 0.63 & 0.0234 & 3.72 & 33 & $2018-1986$ & \multirow{4}{*}{ j; } \\
\hline-1.76 & -0.0585 & 3.33 & 11 & $1996-1986$ & \\
\hline-3.54 & -0.1340 & 3.79 & 11 & $2007-1997$ & \\
\hline 1.54 & 0.0621 & 4.03 & 11 & $2018-2008$ & \\
\hline-0.37 & -0.0082 & 2.21 & 33 & $2018-1986$ & \multirow{4}{*}{$\frac{3}{3}$} \\
\hline-2.11 & -0.0490 & 2.32 & 11 & $1996-1986$ & \\
\hline-5.54 & -0.1158 & 2.09 & 11 & $2007-1997$ & \\
\hline 2.65 & 0.0586 & 2.21 & 11 & $2018-2008$ & \\
\hline-0.15 & -0.0022 & 1.47 & 33 & $2018-1986$ & \multirow{4}{*}{ ' } \\
\hline-0.43 & -0.0065 & 1.51 & 11 & $1996-1986$ & \\
\hline-0.96 & -0.0141 & 1.47 & 11 & $2007-1997$ & \\
\hline 2.26 & 0.0325 & 1.44 & 11 & $2018-2008$ & \\
\hline-0.52 & -0.0073 & 1.40 & 33 & $2018-1986$ & \multirow{2}{*}{ 丁. } \\
\hline-0.70 & -0.0104 & 1.48 & 11 & $1996-1986$ & \\
\hline
\end{tabular}




\section{المجلة الحولية اللعلوم الآنسانية والإمتماعية}

International Journal on Humanities and Social Sciences

website:www.ijohss.com

Email:editor@ijohss.com

العدد (26) أكتوبر 2021

ISSN: $2415-4822$

Volume (26) October 2021

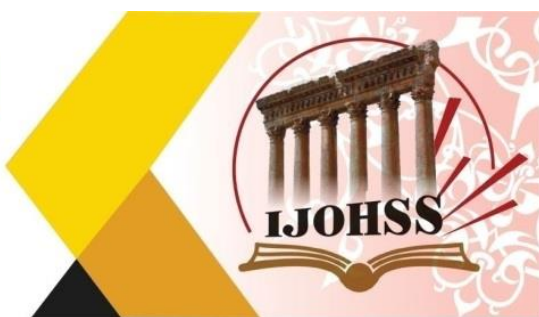

\begin{tabular}{|c|c|c|c|c|c|}
\hline 0.06 & 0.0008 & 1.42 & 11 & $2007-1997$ & \\
\hline 0.35 & 0.0046 & 1.30 & 11 & $2018-2008$ & \\
\hline-0.64 & -0.0097 & 1.52 & 33 & $2018-1986$ & \multirow{4}{*}{ する } \\
\hline 0.87 & 0.0141 & 1.62 & 11 & $1996-1986$ & \\
\hline-2.50 & -0.0385 & 1.54 & 11 & $2007-1997$ & \\
\hline 0.55 & 0.0077 & 1.39 & 11 & $2018-2008$ & \\
\hline-0.33 & -0.0067 & 2.01 & 33 & $2018-1986$ & \multirow{4}{*}{$\begin{array}{l}33 \\
3 \\
3 \\
\overline{3}\end{array}$} \\
\hline-0.14 & -0.0025 & 1.82 & 11 & $1996-1986$ & \\
\hline-0.02 & -0.0005 & 2.54 & 11 & $2007-1997$ & \\
\hline 2.13 & 0.0357 & 1.68 & 11 & $2018-2008$ & \\
\hline-0.04 & -0.0008 & 2.05 & 33 & $2018-1986$ & \multirow{4}{*}{$\begin{array}{l}\sqrt[3]{3} \\
.3 \\
3 \\
3\end{array}$} \\
\hline-1.25 & -0.0254 & 2.04 & 11 & $1996-1986$ & \\
\hline-4.89 & -0.0974 & 1.99 & 11 & $2007-1997$ & \\
\hline 1.05 & 0.0221 & 2.11 & 11 & $2018-2008$ & \\
\hline-0.13 & -0.0033 & 2.51 & 33 & $2018-1986$ & \multirow{4}{*}{$\begin{array}{l}\bar{y} \\
.3 \\
\overline{3} \\
\overline{3}\end{array}$} \\
\hline-0.81 & -0.0208 & 2.56 & 11 & $1996-1986$ & \\
\hline-4.00 & -0.0981 & 2.45 & 11 & $2007-1997$ & \\
\hline 2.67 & 0.0674 & 2.52 & 11 & $2018-2008$ & \\
\hline
\end{tabular}

المصدر : من عمل الباحثة بالاعتماد على موقع المركز الأوروبي للتنبؤات الجوية المتوسطة المدى

ECMWF | ERA Interim, Daily 
المجلة الدولية اللملوم الإسسانية والإمتصاعية International Journal on Humanities and Social Sciences website:www.ijohss.com Email:editor@ijohss.com العدد (26) أكتوبر 2021 ISSN: 2415 - 4822

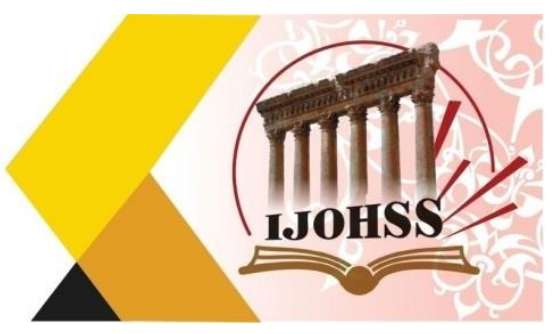

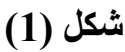

معدلات الثهرية قيم الاوزون للمدة من (1986_1918) في محطات موصل من لأن

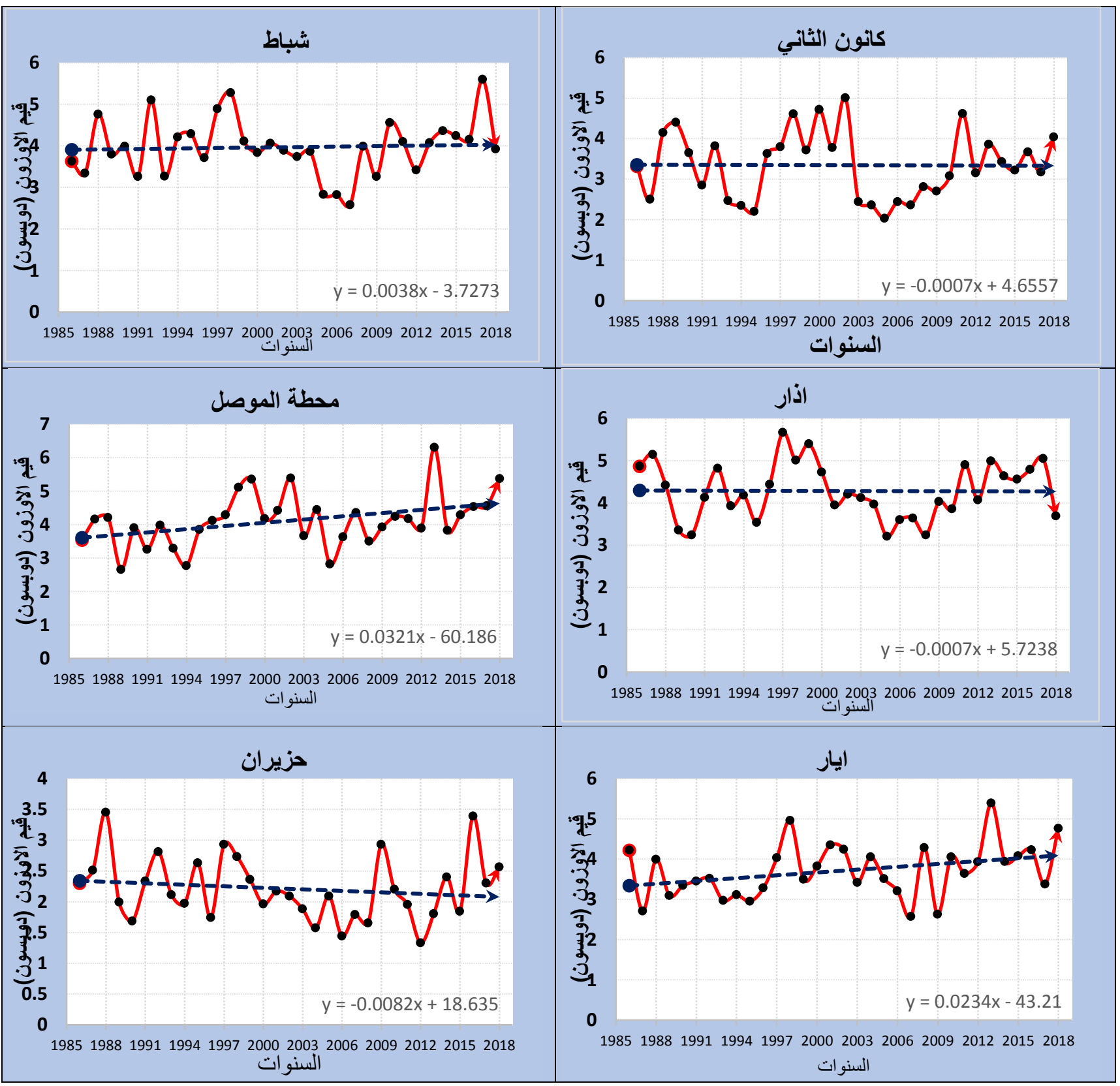


المجلة الدولية اللملوم الإسسانية والإمتصاعية International Journal on Humanities and Social Sciences website:www.ijohss.com Email:editor@ijohss.com العدد (26) أكتوبر 2021 ISSN: 2415 - 4822

Volume (26) October 2021
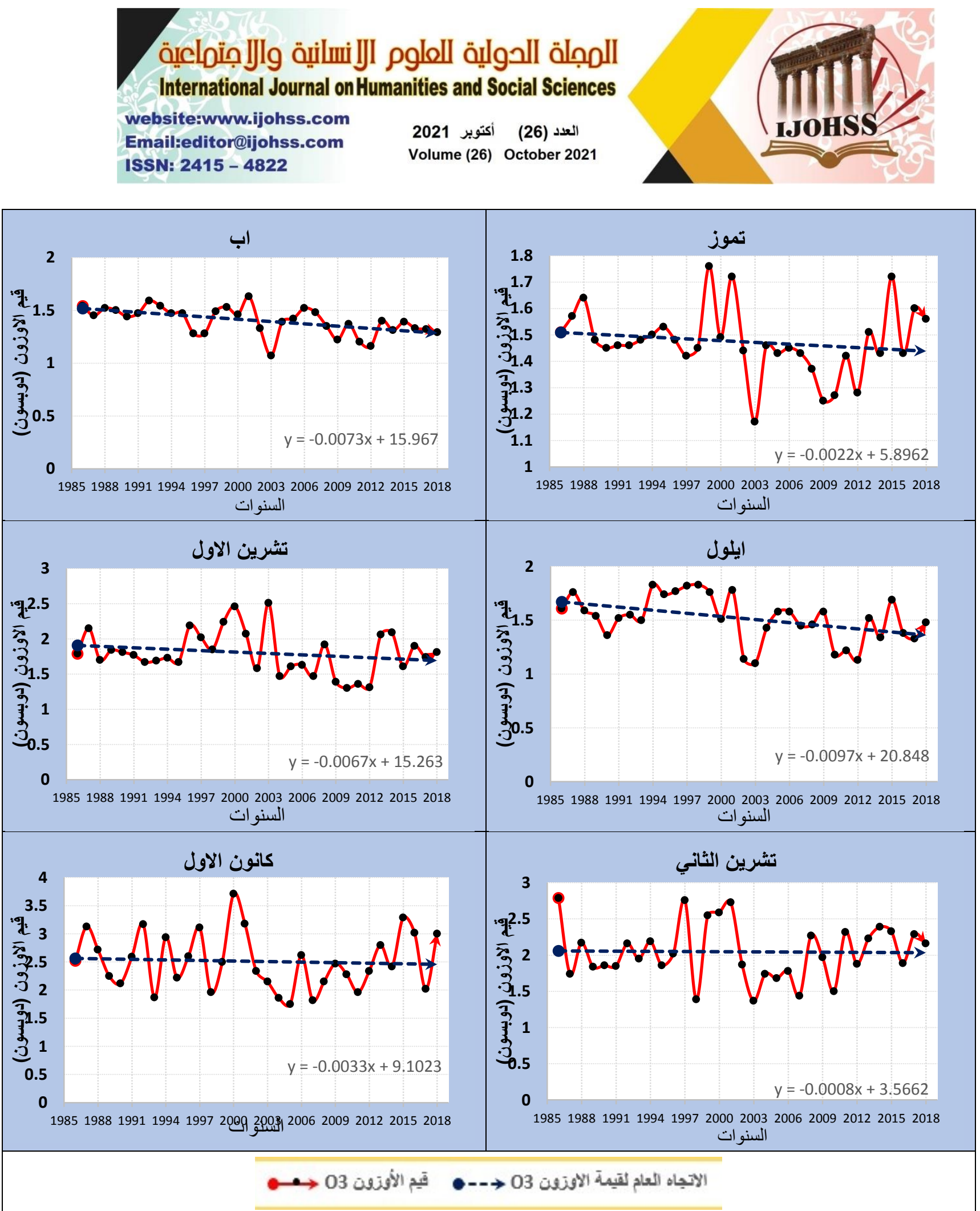

المصدر : الباحثة بالاعتماد على جدول (2). 


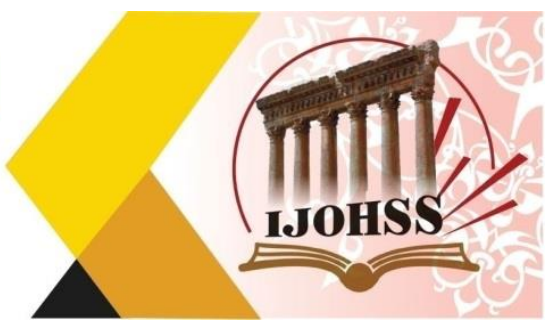

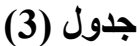

معامل الاتجاه والتغير لقيم الاوزون السنوي للمدة من (32018-1986)

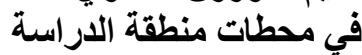

\begin{tabular}{|c|c|c|c|c|c|}
\hline معدل التغير النسبي & الاتجاه & المتوسط & السنو اتد & مدة السلسلة & $\bar{y}: 0$ \\
\hline 0.07 & 0.0019 & 2.72 & 33 & $2018-1986$ & \multirow{4}{*}{$\begin{array}{l}\text { ఫ } \\
\text { ई }\end{array}$} \\
\hline-0.91 & -0.0239 & 2.64 & 11 & $1996-1986$ & \\
\hline-3.96 & -0.1094 & 2.76 & 11 & $2007-1997$ & \\
\hline 1.93 & 0.0532 & 2.75 & 11 & $2018-2008$ & \\
\hline
\end{tabular}

ECMWF | ERA Interim, Daily

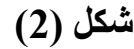

المعدلات السنوية لقيم الاوزون للمدة من (2018_1986) منوصل

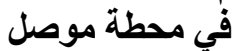

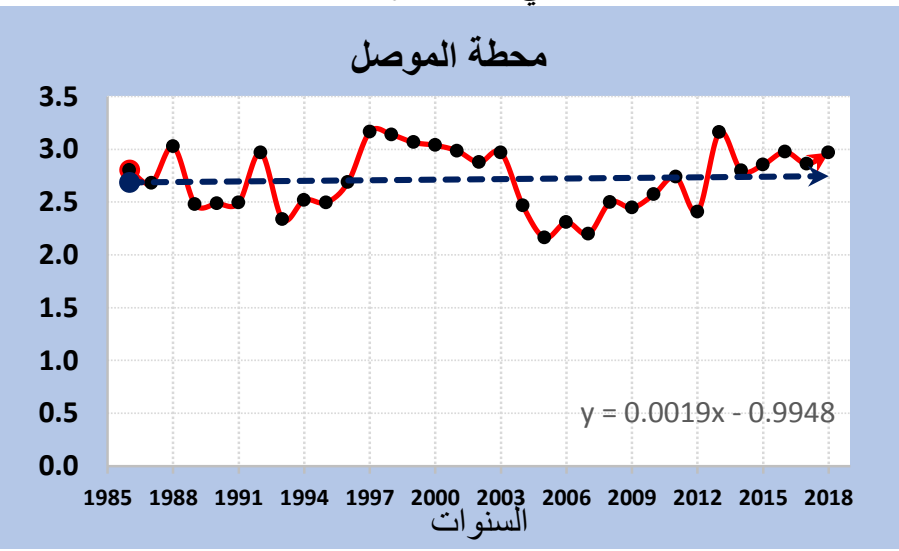

المصدر : الباحثة بالاعتماد على جدول (3).

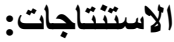

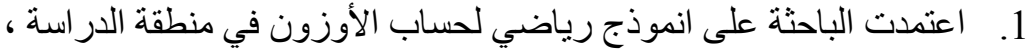

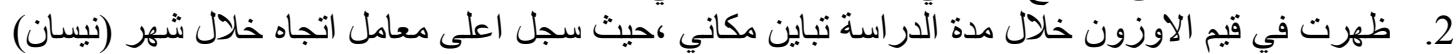

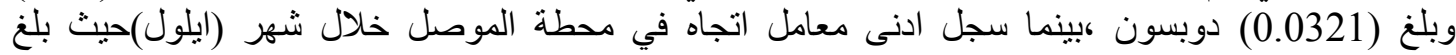

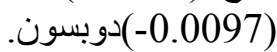

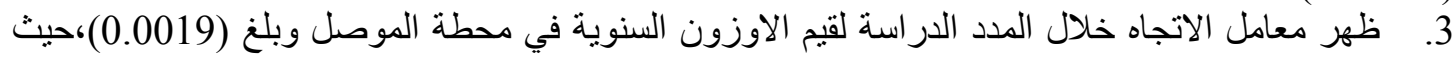

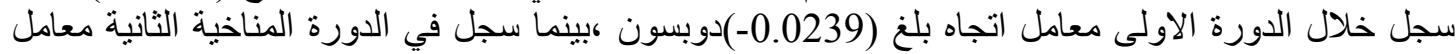

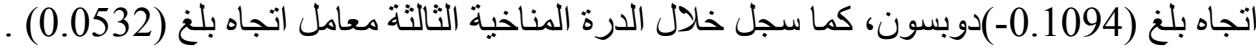

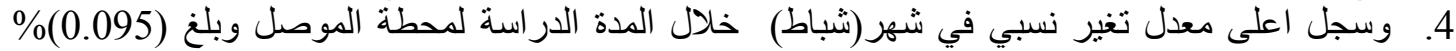
دوبسون ،بينما سجل ادنى معدل تغير نسبي في محطة خلال شهر (ايلول)حيث بلغ (0.64 -)\% دوبسون. 


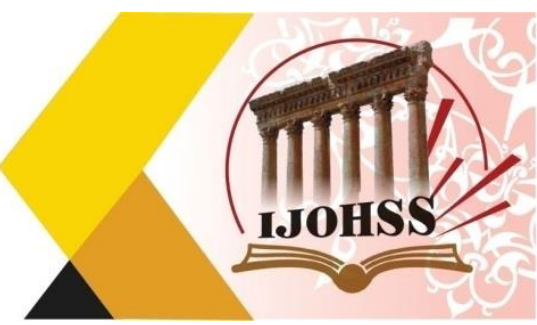

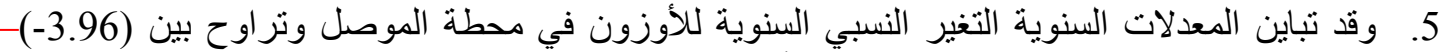
(1.93)\%وحدة دوبسون تم ايجاد تباين مكاني للأوزون في منطقة الدراسة حيث بلغ معدل تغير النسبي خلال

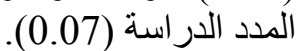

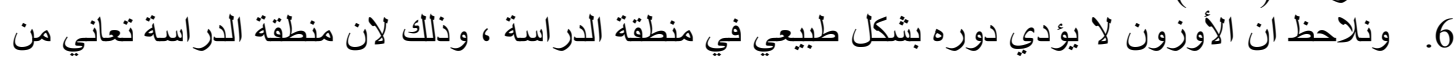

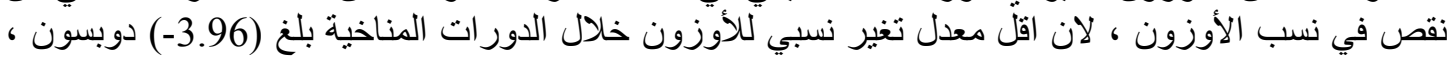

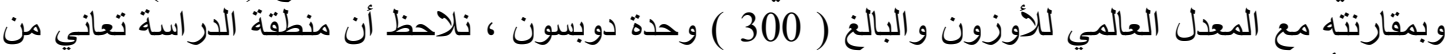

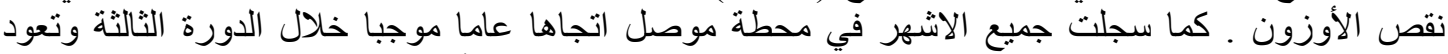

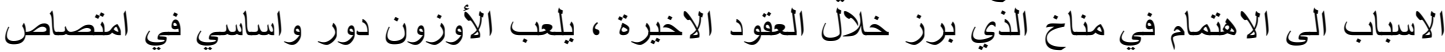

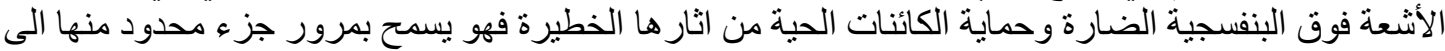

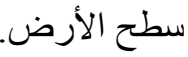

المصادر

1. العبيدي ،مروة خضبر عباس ، ( 2017) ، تحليل التباين المكاني و الزماني لقيم الاشعة الفوق البنفسجية في

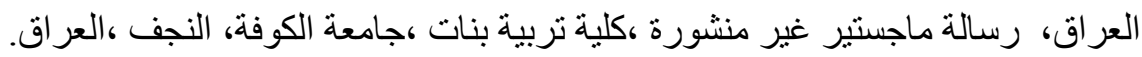

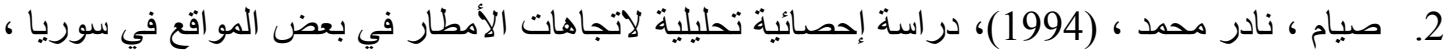
مجلة دمشق ، مجلد (14 ) العدد الثاني. 3. أبو زيد ، محمد صدقه، (2010)، التغير ات الحالية للأمطار السنوية في جنوب محافظة الطائف بالمملكة العربية السعودية ، مجلة علوم الأرصاد و البيئة وزر اعة المناطق الجافة ، جامعة الملك عبد العزيز ، مجلد (21)

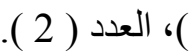
4. عبد القادر عبد العزيز،(2001) ،الطقس و المناخ و الميتورولوجيا، ط82 مطبعة جامعة طنطا ،مصر.

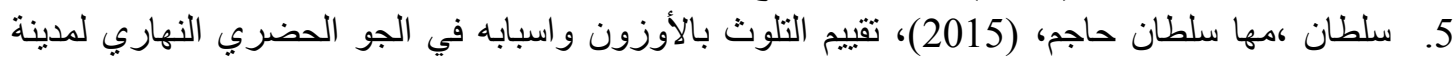

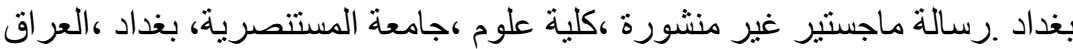

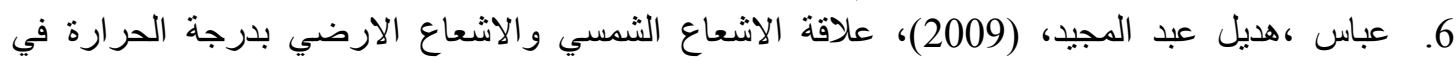

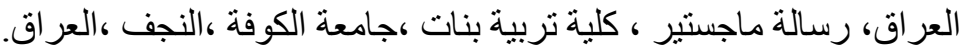

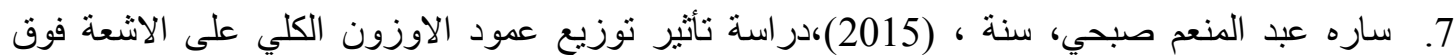

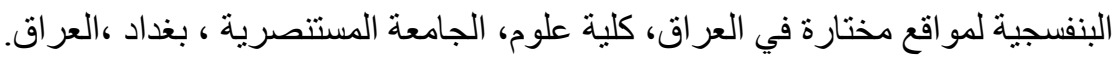

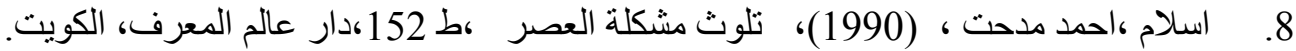

